aneurysm treatment planning. Hence, an automatic computer-based extraction would be beneficial for bleb detection. On the contrary, a universal bleb definition is still missing thus a ground truth for bleb identification is required.

Materials and Methods We conducted an online survey with 40 intracranial aneurysm cases where clinical experts should state whether the intracranial aneurysm possesses none or multiple blebs. In addition, we developed an algorithm to automatically define blebs on the segmented 3D aneurysm surfaces. The algorithm extracts the Gaussian curvature for each vertex of the $3 \mathrm{D}$ aneurysm surface mesh, and divides the surface into regions with positive and negative curvature. Blebs were identified as regions with positive curvature, accompanied by areas of negative curvature.

Results A comparative analysis of our results reveals a strong variation between different raters which further strengthens the need for a bleb definition. The curvature-based automatic detection approach yields two advantages: First, the detection whether a bleb is present or not can support the medical expert when analyzing the aneurysm shape. Second, especially for multiple blebs, the ratings of the medical expert strongly varies in contrast to the automatic bleb detection approach. Hence, a computer-support provides a quantitative and reproducible result.

Conclusion Bleb definition is highly rater-dependent and standardized guidelines are required. Our approach employs curvature of the 3D intracranial aneurysm surfaces for automatic bleb identification to support the clinicians by highlighting lobulated shapes of the aneurysm itself and to reduce interobserver variability between medical experts.

Disclosures D. Behme: None. L. Langolf: None. M. Thormann: None. A. Mpotsaris: None. B. Preim: None. P. Berg: None. S. Saalfeld: None.

\section{E-069 DISCRIMINATION BETWEEN STABLE AND UNSTABLE INTRACRANIAL ANEURYSMS USING IMAGE-BASED BLOOD FLOW SIMULATIONS}

${ }^{1} \mathrm{D}$ Behme*, ${ }^{2} \mathrm{~S}$ Voß, ${ }^{3} \mathrm{~J}$ Korte, ${ }^{1} \mathrm{M}$ Thormann, ${ }^{1} \mathrm{~A}$ Mpotsaris, ${ }^{4} \mathrm{~S}$ Saalfeld, ${ }^{5} \mathrm{G}$ Janiga, ${ }^{3} \mathrm{P}$ Berg. ${ }^{1}$ Neuroradiology, University Clinic Magdeburg, Magdeburg, Germany; ${ }^{2}$ Research Campus Stimulate, University Magdeburg, Magdeburg, Germany; ${ }^{3}$ Research Campus Stimulate; Department of Fluid Mechanics and Technical Flows, University Magdeburg, Magdeburg, Germany; ${ }^{4}$ Research Campus Stimulate; Department of Simulation and Graphics, University Magdeburg, Magdeburg, Germany; ${ }^{5}$ Department of Fluid Dynamics and Technical Flows, University Magdeburg, Magdeburg, Germany

\subsection{6/neurintsurg-2021-SNIS.164}

Introduction/Purpose The discrimination between hemodynamically stable and unstable intracranial aneurysms remains challenging. Recent studies reveal the potential existence of intraaneurysmal velocity fluctuations, which appear at high frequency. Since these flow disturbances might promote the vessel wall remodeling process, indications regarding the occurrence of such phenomena are desired.

Materials and Methods To quantify unstable hemodynamics, image-based blood flow simulations were carried out in a ruptured aneurysm at the posterior inferior cerebellar artery.

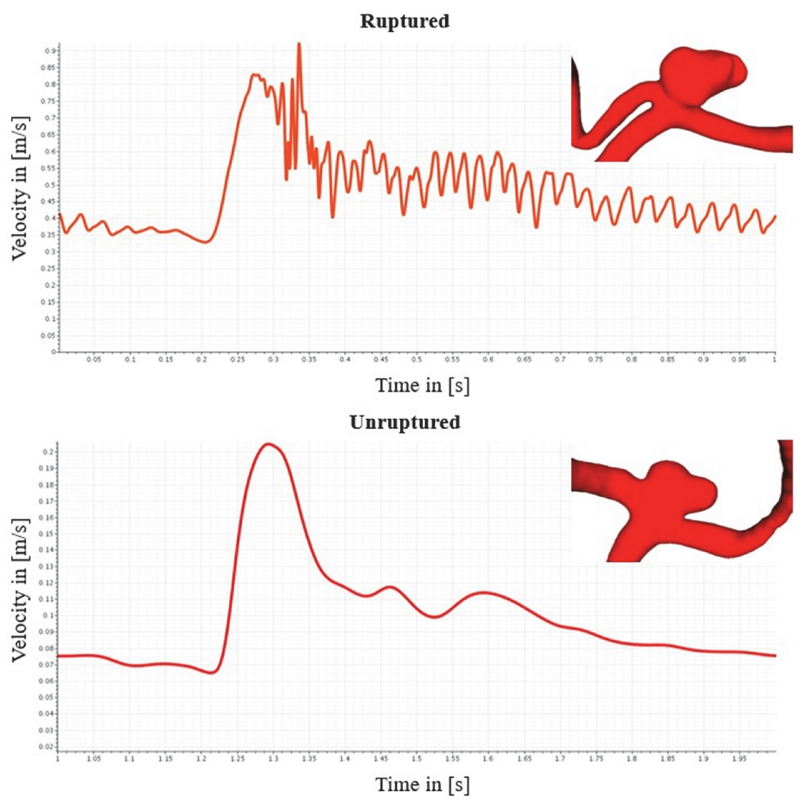

Abstract E-069 Figure 1 Intra-aneurysmal velocity waveform for the ruptured (top) and reference unruptured (bottom) intracranial aneurysm. Notice the high-frequency fluctuations throughout the whole cardiac cycle for the ruptured case

Since the exact rupture site could be identified during the angiographic imaging, local flow analysis was feasible. Beside the evaluation of time-varying velocity fluctuations, shear stress distributions and vortex generations were analyzed. Furthermore, the existing spectral flow entropy was assessed using proper orthogonal decomposition. Finally, all simulations were repeated in an unruptured aneurysm at the middle cerebral artery, which was similar with respect to size and shape.

Results The high-resolution blood flow simulations reveal the existence of complex flow patterns in combination with highfrequency fluctuations. These predominantly occur after the peak-systolic inflow and maintain in the diastolic phase. Furthermore, the wall shear stress distribution demonstrates a strong oscillatory behavior emphasizing the unstable character of the flow. The calculation of the spectral entropy resulted in a value of 0.76 , which was associated with highly disturbed flow in advance. In contrast, these observations are absent in the reference simulation of the unruptured aneurysm. Specifically, stable flow patterns are visible and a low spectral entropy of 0.12 was calculated (a value of 0 represents steady flow conditions).

Conclusion The presence of high-frequency fluctuations and increased spectral entropy could be a potential biomarker for the discrimination between hemodynamically stable and unstable intracranial aneurysms. Since strong deviations were detectable in aneurysms with similar shape and location, stronger focus on the surrounding vasculature (e.g., with respect to the angle of proximal and distal branches) is suggested.

Disclosures D. Behme: None. S. Voß: None. J. Korte: None. M. Thormann: None. A. Mpotsaris: None. S. Saalfeld: None. G. Janiga: None. P. Berg: None. 